\title{
Statistical Mechanics Built on Sand
}

\author{
The results of new experiments validate the idea that there exists a \\ statistical mechanics framework for granular materials.
}

\author{
By Adrian Baule
}

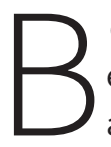

each sand, sugar, and coffee powder are just three examples of life's ubiquitous granular materials, which are materials that are composed of small particles or grains. Despite this ubiquity, a fundamental understanding of the properties of these materials has, so far, remained elusive. Now, Yujie Wang at Shanghai Jiao Tong University, China, and collaborators take a step toward developing that understanding by performing experiments that validate a statistical mechanics framework for granular systems [1]. Their results could help researchers in determining novel methods for predicting the properties of granular materials.
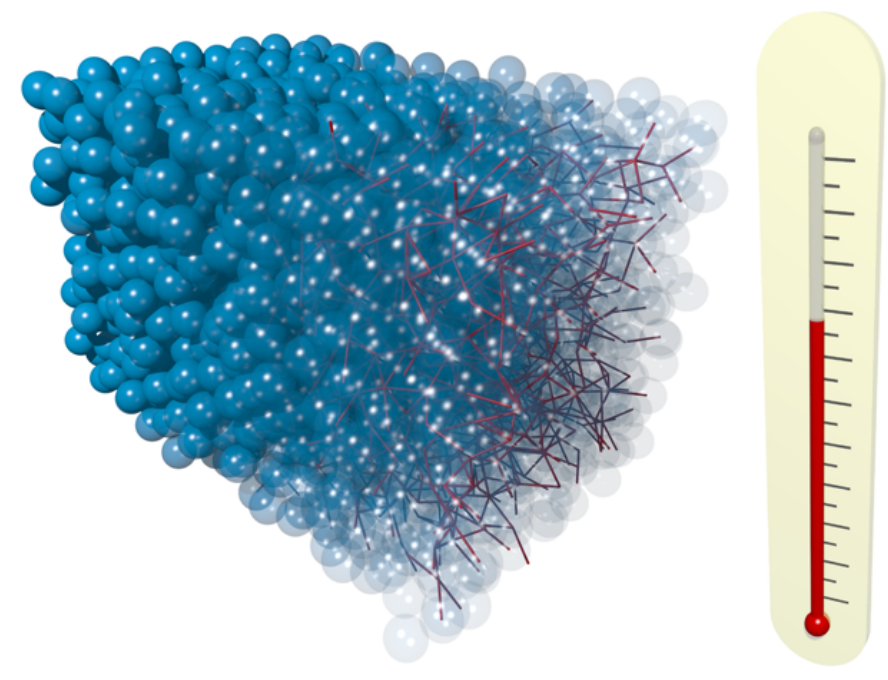

Figure 1: New experiments that measure the volume statistics of a container full of plastic beads validate a statistical mechanics framework for granular materials.

Credit: Y. Wang/Shanghai Jiao Tong University
Granular materials exhibit similar behaviors to other multiparticle systems, such as gases, liquids, and solids. For example, like liquids, granular materials can flow when they are poured, and, like solids, they can support stresses when in a jammed state. The macroscopic observables of a granular system, such as its packing density, can be reproduced by controlling only a few of the system's parameters, highlighting that it is governed by statistical principles. Gases, liquids, and solids at thermal equilibrium are also governed by statistical principles and can be successfully described by the theory of equilibrium statistical mechanics. In contrast, establishing a similarly comprehensive statistical theory of granular systems has been a long-standing problem.

In equilibrium statistical mechanics, the central observable is the system's total energy. This energy relates to both the entropy of the system (the number of microstates accessible at a given energy) and the probability that the system sits in a configuration with a particular energy. For granular systems, however, the total energy is either unconserved (as is the case in systems made up of frictional, dissipative particles) or irrelevant (as is the case for systems made up of frictionless, hard particles), and thus energy is unsuitable as a state variable.

One promising state variable for granular materials is the system's volume. More than three decades ago, the late Sam Edwards of the University of Cambridge and colleagues hypothesized that volume could play the role of energy for granular systems. They showed that they could formulate a statistical-mechanics-based theory, using the system's volume, that contained concepts such as granular entropy, "compactivity" (a temperature-like variable), and a canonical 
volume ensemble that determines the probability of observing a configuration with a specific volume [2, 3]. This framework has received much scrutiny. But directly testing the theory's predictions via experiments or by simulations has been challenging [4].

The challenge arises because the properties of a granular system typically depend on the preparation protocol. It matters, for example, whether grains create a jammed aggregate after being poured into a container under gravity or whether they are compactified from some loose configuration by moving the container walls. The final packing structure can contain remnants of the initial state and of the preparation details, neither of which are accounted for in the theory. In addition, it is difficult to faithfully reproduce underlying conditions of the theory, such as the canonical ensemble condition, which requires that volume be exchanged between the system and a surrounding "heat bath." Wang and colleagues have now resolved these issues in their new experiments.

The team's work follows in the footsteps of earlier experiments that mimicked the effect of randomizing heat fluctuations by repeatedly "exciting" the system $[5,6]$. Under such excitations, the system can reach one of a number of stationary states that has a specific packing density and that is independent of how the system was prepared. Researchers have interpreted these stationary states as equilibrium-like states that should, in principle, be describable by a statistical mechanics formalism.

In the new experiments, Wang and colleagues studied a granular material consisting of several-mm-diameter 3D-printed plastic beads held in a container. For different iterations of the experiment, the team used beads with different roughnesses, allowing them to study the effect of friction on the packing statistics. They excited the material by periodically tapping the container and monitored the bead configurations using $\mathrm{x}$-ray tomography.

The Edwards framework considers the volume of a given system, which fluctuates under an exchange of volume with its surroundings. Wang and colleagues instead consider "subsystems" within the container, with a subsystem being defined as a spherical region of fixed diameter around a particle, whose volume can be precisely determined. These subsystems are large enough that they remain uncorrelated and can thus be considered as independent realizations of the same system for which ensemble statistics can be established.

Using this subsystem idea, Wang and colleagues found that the probability distribution of the associated volumes follows the functional form of Edwards' canonical volume ensemble. They were also able to calculate the system's compactivity and its granular entropy using two different methods. Surprisingly, they found that the relationship between compactivity and tapping intensity is independent of friction, indicating that different but identically excited granular systems assume the same compactivity. In the context of equilibrium statistical mechanics, this behavior corresponds to that seen when two systems in contact with the same heat bath achieve the same temperature-a manifestation of the zeroth law of thermodynamics.

The data of Wang and colleagues supports the validity of a granular zeroth law of thermodynamics within the experimental uncertainty, which contrasts with a previous finding that the zeroth law did not hold for a 2D compaction experiment involving frictional disks [7]. This discrepancy could come from the different packing protocols used in the two sets of experiments or from subtle differences in the way the compactivity was measured. The new data can also be summarized in a phase diagram, which characterizes packings in terms of the packing density, the friction coefficient, and the average number of contacts per particle, extending previous results obtained from mean-field theory [8]. Different friction coefficients map out different curves in this phase diagram, but, under suitable rescaling, the curves collapse onto a single curve, indicating universal behavior of the frictional packings.

The new results add to a growing body of work that supports the validity of statistical mechanics approaches for granular materials $[9,10]$. However, using these approaches to make specific calculations remains highly challenging [4]. Much more work, both theoretical and experimental, is needed to fully exploit the explanatory power of granular statistical mechanics for real-world applications.

Adrian Baule: School of Mathematical Sciences, Queen Mary University of London, London, United Kingdom 


\section{REFERENCES}

1. Y. Yuan et al., "Experimental test of the Edwards volume ensemble for tapped granular packings," Phys. Rev. Lett. 127, 018002 (2021).

2. S. F. Edwards and R. B. S. Oakeshott, "Theory of powders," Physica A 157, 1080 (1989).

3. A. Mehta and S. F. Edwards, "Statistical mechanics of powder mixtures," Physica A 157, 1091 (1989).

4. A. Baule et al., "Edwards statistical mechanics for jammed granular matter," Rev. Mod. Phys. 90, 015006 (2018).

5. E. Nowak et al., "Density fluctuations in vibrated granular materials," Phys. Rev. E 57, 1971 (1998).

6. M. Schröter et al., "Stationary state volume fluctuations in a granular medium," Phys. Rev. E 71, 030301 (2005).

7. J. G. Puckett and K. E. Daniels, "Equilibrating temperaturelike variables in jammed granular subsystems," Phys. Rev. Lett. 110, 058001 (2013).

8. C. Song et al., "A phase diagram for jammed matter," Nature 453, 629 (2008).

9. S. Martiniani et al., "Numerical test of the Edwards conjecture shows that all packings are equally probable at jamming," Nat. Phys. 13, 848 (2017).

10. E. S. Bililign et al., "Protocol dependence and state variables in the force-moment ensemble," Phys. Rev. Lett. 122, 038001 (2019). 